\title{
SHORT REPORT: ESTABLISHING NORMAL HEARING FOR INFANTS WITH THE AUDITORY STEADY-STATE RESPONSE
}

\author{
De Wet Swanepoel* (corresponding author) and Karen Steyn
}

Department of Communication Pathology

University of Pretoria

\begin{abstract}
This study investigated the use of the dichotic multiple frequency ASSR technique for characterising normal hearing in a group of infants. A descriptive research design was implemented to describe ASSR thresholds obtained in 10 normal hearing infant ears ( 3 male, 2 female participants) between the age of 3 and 8 weeks. Normal hearing was controlled for by conducting a DPOAE screening test on all ears and ensuring no risk factors for hearing loss were present. Results indicated mean ASSR thresholds at $0.5,1,2$, and $4 \mathrm{kHz}$ to vary between 30 and $37 \mathrm{~dB}, \pm 8-11 \mathrm{~dB}$ within a range of $20-50 \mathrm{~dB} \mathrm{HL}$. Eighteen percent of ASSR thresholds were obtained at $20 \mathrm{~dB}, 45 \%$ were obtained at $30 \mathrm{~dB}$, and $38 \%$ were obtained at elevated levels of 40 and $50 \mathrm{~dB}$. The recorded dichotic multiple frequency ASSR thresholds for infants with normal hearing were within the mild to moderate hearing loss range which makes differentiating between less severe degrees of hearing loss and normal hearing difficult. Until future research has been conducted, caution must be practiced when interpreting ASSR thresholds below $60 \mathrm{~dB}$ in young infants and additional techniques such as the ABR must be used to cross-check results.
\end{abstract}

Key words: Auditory Steady State Response, diagnostic audiology, paediatric audiometry, objective audiometry, infant hearing, electrophysiological audiometry.

\section{INTRODUCTION}

The importance of early intervention, to ensure optimal outcomes for infants with hearing loss, is firmly established and clearly evidenced in the increasing number of newborn hearing screening programmes implemented world-wide (YoshinagaItano, 2003). The successful outcomes for infants with hearing loss is, however, foremost dependent on the accurate characterisation of hearing ability as all subsequent intervention practices build on this cornerstone (Gorga, 1999). Since the hearing of young infants is not easily evaluated by conventional behavioural audiometric measures, diagnostic assessments utilising electrophysiologic techniques such as the Auditory Brainstem Response (ABR) is used to estimate hearing thresholds.

The ABR has served as the gold standard electrophysiological procedure for determining hearing loss in neonates and young infants incapable of providing conditioned auditory responses to sounds for the last three decades. It is only recently that another clinical instrument for estimating hearing thresholds in infants, the Auditory Steady-State Response (ASSR), has become available (Swanepoel \& Hugo, 2004). The ASSR promises a number of advantages over the ABR such as frequency specific threshold estimation similar to pure tone audiometry; elevated maximum stimulation levels up to $120 \mathrm{~dB}$ HL allowing differentiation between severe and profound hearing losses; objective threshold determination by reliable statistical techniques; aided threshold estimations with less stimulus and output distortion; and time efficient testing using the dichotic multiple frequency technique, which allows assessment of multiple frequencies in

\footnotetext{
* Department of Communication Pathology

University of Pretoria

South Africil

Pretori:

00012

Tel: 27124202949

Fax: 27124203517

E-mail: dewet.swancpocl@up.ac.za
}

both ears simultaneously (Picton et al., 1998; Rance, Dowell, Rickards, Beer \& Clark, 1998; Swanepoel \& Hugo, 2004; Swanepoel, Hugo \& Roode, 2004).

Rickards et al. (1994) were the first to demonstrate that ASSRs can be successfully recorded from normal full-term sleeping neonates. Subsequent ASSR studies in neonates and young infants using a single frequency technique have consistently demonstrated its effectiveness in characterising moderate to profound hearing losses with increasingly accurate estimations for more severe hearing losses (Cone-Wesson, Dowell, Tomlin, Rance \& Ming, 2002; Rance et al., 1998; Rance \& Briggs, 2002; Rance \& Rickards, 2002; Swanepoel \& Hugo, 2004). The closer correlation between ASSR and behavioural thresholds for severe and profound hearing losses has primarily been attributed to the recruitment effect (Rance et al.! 1998). Few reports are however available for ASSR threshold estimations in normal hearing infants and no reports on ASSR thresholds for mild hearing losses are available to date (John, Brown, Muir \& Picton, 2004; Luts, Desloovere, Kumar, Vandermeersch \& Wouters, 2004; Rance \& Rickards, 2002).

Rance and Rickards (2002) reported typical ASSR।thresholds for normal hearing neonates and babies to be between 30 and $40 \mathrm{~dB}$ HL compared to mean ASSR thresholds between 25 and $40 \mathrm{~dB}$ HL reported by Rickards et al. (1994). A more recent study by John et al. (2004) reported average ASSR thresholds for the $500 \mathrm{~Hz}$ stimulus in infants in their first few days of life to be approximately $40 \mathrm{~dB}$ HL. The average thresholds at 1000 , 2000 , and $4000 \mathrm{~Hz}$ were reported to be between 10 to $15 \mathrm{~dB}$ better and the data suggest an improvement in thresholds, of approximately $10 \mathrm{~dB}$ within the first few months of life except at $500 \mathrm{~Hz}$ (John et al., 2004). It was also concluded that the amplitude of the response was significantly increased when mixed modulations (frequency and amplitude) were used and that responses were more easily detected in infants older than three weeks of age relative to the first few days after birth (John et al., 2004). 
The ASSR has already demonstrated exciting benefits but more comprehensive descriptions of ASSR threshold estimations for young infants, especially for the dichotic multiple frequency technique, are required to ensure it is used in a scientifically accountable manner (Luts et al., 2004). The dearth of ASSR reports for normal hearing neonates and infants using the dichotic multiple frequency ASSR and preliminary evidence of ASSR threshold changes over the first few months has provided the rationale for the current investigation. In this study, dichotic multiple frequency ASSR thresholds are investigated in 10 normal hearing ears of infants younger than two-months.

\section{METHOD}

The institutional review board at the University of Pretoria approved this project before any data were collected. Informed consent from the parent/legal guardian was also obtained before any testing was conducted.

\section{Participants}

A sample of 5 participants (10 ears) with normal hearing was enrolled using a convenience sampling method. Three of the participants were male. All participants were between the age of 3 and 8 weeks with a mean age of 5 weeks. Normal hearing was controlled for by ascertaining that no risk indicators for hearing loss, as specified by the JCIH Year 2000 position statement (JCIH, 2000), were present and all ears passed a DPOAE screen. Although OAE testing is only a test of pre-neural integrity of the outer hair cells in the cochlea, normal hearing may be inferred with a high degree of confidence based on an OAE pass result since OAE screening has evidenced consistent specificity rates above 97\% (Lutman, 2000; Prieve \& Stevens, 2000). Cases of auditory neuropathy, which may present with OAE's and absent auditory responses from the auditory nerve or brainstem, was controlled for by ensuring that no risks for hearing loss including admittance to the NICU was present in any of the participants. Auditory neuropathy has been shown to be highly correlated with risk factors especially those associated with admittance to the NICU (Rapin \&, Gravel, 2003; Sininger, 2002).

The risk factors were controlled for by an interview with the caregiver. The OAE screening was conducted using a Biologic ABaer Cub screener (Ver 2.9.0) with a Distortion Product OAE screening protocol requiring three out of four passes for frequencies between 1 to $5 \mathrm{kHz}$. All infants were tested during natural sleep and in certain instances more than one appointment was scheduled to gather all the necessary information.

\section{Stimuli}

ASSRs were evoked using a dichotic multiple frequency technique stimulating both ears simultaneously with four carrier frequencies per ear. Test stimuli were $0.5,1,2$, and $4 \mathrm{kHz}$ tones modulated in amplitude and frequency with a relative AM/FM phase difference of $90^{\circ}$. The tones were $20 \%$ frequency modulated and $100 \%$ amplitude modulated at $82,84,87$, and $89 \mathrm{~Hz}$ respectively for the $0.5,1,2$, and $4 \mathrm{kHz}$ tones in the left ear and 91,94 , 96 , and $99 \mathrm{~Hz}$ for the $0.5,1,2$, and $4 \mathrm{kHz}$ tones respectively in the right ear. These modulation rates were according to the default specifications of the Biologic Corporation MASTER system (version 1.8). Modulation rates in excess of $70 \mathrm{~Hz}$ were used to ensure that a satisfactory signal to noise ratio would exist for detection of responses during sleep or sedation. Test stimuli were presented through EAR 3A insert earphones calibrated in hearing level. The stimuli were separately calibrated for each frequency using pure tones according to the AS 1591.2 standard. All measurements were made with a Brüel and Kjaer sound level meter model Investigator 2260 , an artificial ear type 4152 and a microphone type 4144 .

\section{Recordings}

All ASSR recordings were obtained in a single-walled sound booth within a sound treated room.

ASSR Measurements: ASSR assessments were performed on the Biologic MASTER system (Version 1.8) using a dichotic multiple frequency technique. This dichotic multiple frequency technique implies that multiple frequencies were evaluated in both ears simultaneously. This type of simultaneous stimulation has been demonstrated to be a time-efficient way of determining ASSR thresholds (Dimitijevic et al., 2002; Perez-Abalo et al., 2001). Electrode discs of $\mathrm{Ag} / \mathrm{AgCI}$ were fixed with electrolytic paste to the scalp at $\mathrm{Cz}$ (Active), midline posterior neck (Reference), and Fpz (Ground). All electrode impedances were below $5 \mathrm{kOhm}$ at $10 \mathrm{~Hz}$ and the inter-electrode impedance values were kept below $3 \mathrm{kOhm}$. The bioelectric activity was amplified and analog filtered using a 3 to $300 \mathrm{~Hz}$ bandpass filter. A maximum of 32 sweeps containing 16 epochs each was recorded per trial. Each epoch was $1.024 \mathrm{~s}$ and a complete sweep lasted 16.384 s. The electrophysiological recording was converted using a Fast Fourier Transform (FFT) after each sweep. The presence of a response was determined using a F-ratio comparing the Fast Fourier components at the stimulus modulation frequencies to the 120 adjacent frequencies ( 60 bins above and 60 bins below the frequency) to determine if the difference was significantly different $(\mathrm{p}<.05)$ from the background noise. If a sweep contained more than $40 \mu \mathrm{V}$ electrophysiological noise it was rejected. A recording was halted once a pre-set probability of $95 \%$ response significance was achieved after averaging at least five sweeps, or when a statistically significant probability value could not be achieved within 32 sweeps $(524,29 \mathrm{~s})$. The threshold-seeking procedure utilised a $10 \mathrm{~dB}$ intensity step. A recent study indicated that a smaller $5 \mathrm{~dB}$ step compared to a $10 \mathrm{~dB}$ step did not make the estimate more precise and increased the recording time which negatively influences ASSR recordings (Luts \& Wouter, 2004). The initial stimulation intensity was $40 \mathrm{~dB} \mathrm{HL}$. If a significant response was not obtained in both ears at this intensity, the intensity was increased until a significant response was obtained in both ears. Once a significant response was obtained for both ears, the intensity was lowered to obtain a threshold in both ears. Threshold was taken as the lowest intensity where a response was elicited with no response found at a lower level.

\section{RESULTS}

ASSR thresholds were obtained for all frequencies evaluated in the sample of infants. Table 1 indicates the mean, standard devia-

Table 1. ASSR thresholds $(n=40)^{*}$ for 10 normal hearing infant ears

\begin{tabular}{|c|c|c|c|c|}
\hline $\mathbf{k H z}$ & $\begin{array}{c}\text { Mean } \pm \text { SD } \\
\text { All ears } \\
\text { (dB HL) }\end{array}$ & $\begin{array}{c}\text { Mean } \pm \text { SD } \\
\text { Left ears } \\
\text { (dB HL) }\end{array}$ & $\begin{array}{c}\text { Mean } \pm \text { SD } \\
\text { Right ears } \\
\text { (dB HL) }\end{array}$ & $\begin{array}{c}\text { Range } \\
\text { (dB) }\end{array}$ \\
\hline 0.5 & $37 \pm 8$ & $36 \pm 9$ & $38 \pm 8$ & $30-50$ \\
1 & $34 \pm 10$ & $34 \pm 11$ & $34 \pm 9$ & $20-50$ \\
2 & $34 \pm 11$ & $36 \pm 11$ & $32 \pm 11$ & $20-50$ \\
4 & $30 \pm 11$ & $32 \pm 8$ & $30 \pm 14$ & $20-50$ \\
\hline
\end{tabular}

* Number of ASSR thresholds recorded for the sample of ears 
tion, and range of the 40 ASSR thresholds obtained for the sample of ears (4 frequencies $x 10$ ears).

The mean ASSR thresholds for the entire sample varied between 30 and $37 \mathrm{~dB}$ with the closest approximation to normal hearing levels at $4 \mathrm{kHz}$ and the furthest at $0.5 \mathrm{kHz}$. Standard deviations varied between 8 and $11 \mathrm{~dB}$ with the smallest deviation at $0.5 \mathrm{kHz}$ and the largest at 2 and $4 \mathrm{kHz}$.

The ASSR thresholds for the sample ranged between 20 and $50 \mathrm{~dB}$. Figure 1 indicates the distribution of the ASSR thresholds for the sample of ears.

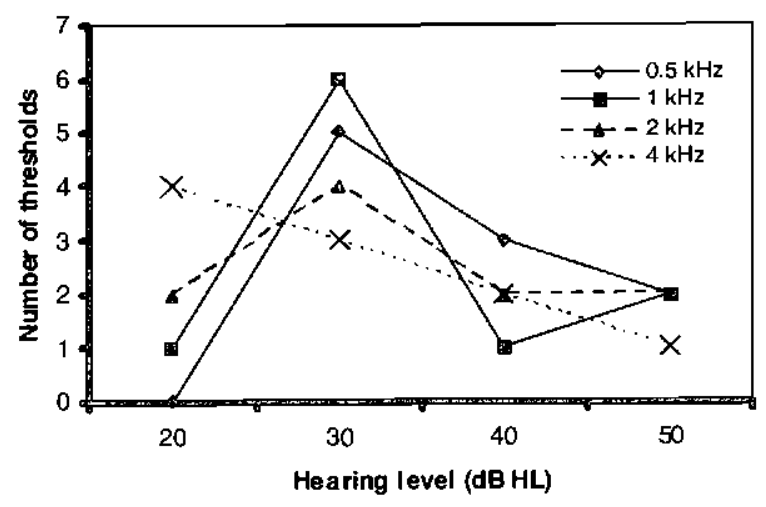

Figure 1. Distribution of ASSR thresholds $(n=40)$

Almost half $(45 \%)$ of ASSR thresholds were obtained at $30 \mathrm{~dB}$ whilst only $18 \%$ were obtained at $20 \mathrm{~dB}$. A large proportion (38\%) of ASSR thresholds for this sample of normal hearing infants were obtained at elevated levels of 40 and $50 \mathrm{~dB}$. Half (50\%) of $0.5 \mathrm{kHz}$ thresholds and 30 to $40 \%$ of thresholds for the higher frequencies ( 1 to $4 \mathrm{kHz}$ ) were obtained at these elevated intensities ( 40 to $50 \mathrm{~dB}$ ).

Figure 2 represents the thresholds of two ASSR recordings illustrating the ear with the closest approximation and the ear with the furthest approximation of normal hearing levels for pure tones ( 0 to $15 \mathrm{~dB}$ ) (Goodman, 1965; Clark, 1981).

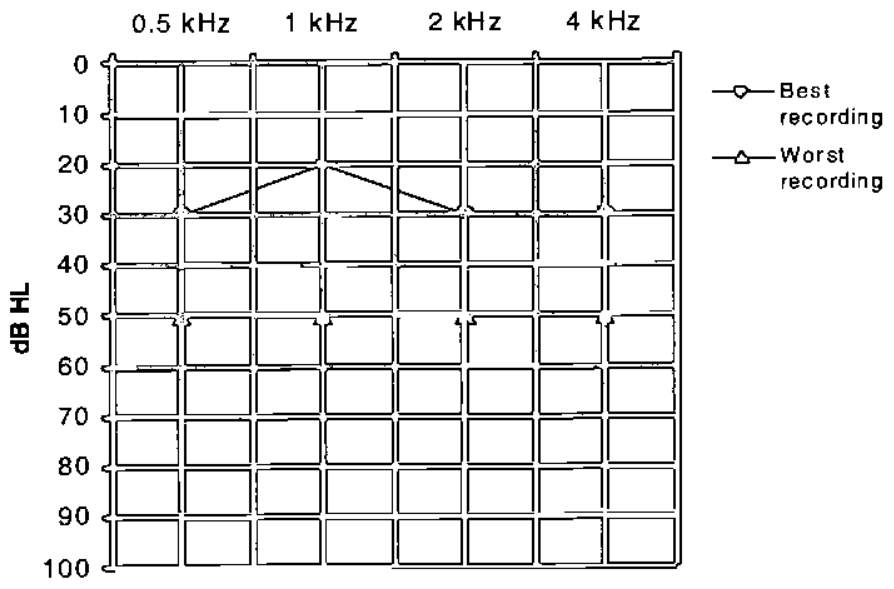

Figure 2. Best and worst ASSR estimation of normal hearing in sample of infant ears

According to Clark's (1981) modification of Goodman's (1965) scale for classifying hearing loss according to pure tone thresholds, the ASSR thresholds in the ear with the best approximation of normal hearing presents with a mild hearing loss (26 to $40 \mathrm{~dB}$ ) whilst the ear with the worst approximation presents with

\section{DISCUSSION}

The accuracy of ASSR thresholds are measured by how closely they approximate pure tone behavioural thresholds which provide the gold standard for hearing status. The results of the current study indicate that the majority $(83 \%)$ of ASSR thresholds for young infants with normal hearing were within the mild to moderate hearing loss range according to the scale of hearing loss severity for pure tone thresholds (Clark, 1981). This finding is in agreement with previous studies conducted using the single frequency ASSR technique in which the majority of thresholds for normal hearing infants were reported to be between 25 and 40 dB HL (Rance \& Rickards, 2002; Rickards et al., 1994). Studies reporting ASSR thresholds for normal hearing infants using the dichotic and monotic multiple frequency technique also suggest similar average values between 25 and $40 \mathrm{~dB}$ (John et al., 2004; Lins et al., 1996). John et al. (2004) indicated that after the first three weeks of life the ASSR thresholds may improve by $10 \mathrm{~dB}$. The current study investigated infants older than three weeks and a $10 \mathrm{~dB}$ improvement in the 25 to $40 \mathrm{~dB}$ average was not observed. A smaller intensity step of $5 \mathrm{~dB}$ compared to $10 \mathrm{~dB}$ may have resulted in closer estimates of behavioural thresholds although a recent study indicated that a $5 \mathrm{~dB}$ step size did not make the estimate more precise but increased testing time by up to 60 minutes (Luts \& Wouter, 2004).

The general trend of the current research evidence does however suggest that ASSR thresholds improve within the first several months of life for infants with normal hearing (John et al., 2004; Lins et al., 1996; Rance \& Rickards, 2002). This improvement in hearing within the first few weeks means that normal hearing infants present with elevated ASSR thresholds as seen in the current study, which makes differentiating between mild-tomoderate hearing losses and normal hearing in young infants very difficult. Although Rance and Briggs (2002) concluded that the ASSR can reliably quantify hearing loss in infants, their study cohort only included infants with moderate to profound hearing loss. No studies have demonstrated the ability of the ASSR to differentiate between mild and mild-to-moderate hearing losses and normal hearing in young infants. Caution is therefore necessary when interpreting ASSR threshold data up to $50 \mathrm{~dB}$ HL to ensure that appropriate diagnoses are made which clearly differentiates between milder hearing losses and normal hearing in infants. The importance of correctly characterising hearing loss in such cases have been indicated by several studies which demonstrate that mild or moderate and even minimal degrees of hearing loss can lead to significant delays in language development and academic achievement in children (Bess, Dodd-Mụrphy \& Parker, 1988; Carney \& Moeller, 1998; Davis, Elfenbein, Schum \& Bentler, 1986).

To improve ASSR estimations, regression formulae to predict behavioural thresholds from ASSR threshold data were developed by Rance and colleagues (Rance, Rickards, Cohen, De Vidi \& Clark, 1995; Rance \& Rickards, 2002) based on threshold data from a large cohort of participants with varying degrees of hearing loss. These formulae, used by the GSI Audera system to predict actual behavioural thresholds, has proved useful in making confident estimations in infants with ASSR, thresholds above $60 \mathrm{~dB}$ (Rance and Briggs, 2002). The algorithms have not yet however demonstrated the capability to clearly differentiate between slight, mild and mild-to-moderate hearing losses and normal hearing in young infants. This lack of research evidence supporting the efficacy of ASSR estimations of mild hearing loss emphasises the need for caution when interpreting ASSR thresh- 
olds below $60 \mathrm{~dB}$ HL in young infants. In such cases, additional testing, using the ABR with click and tone burst stimuli, is required to cross-check the ASSR data (Swanepoel, Schmulian \& Hugo, 2004).

In conclusion, this study indicated that dichotic multiple frequency ASSR thresholds for infants with normal hearing are recorded within the mild to moderate range of hearing loss. These elevated ASSR thresholds suggest difficulties differentiating between normal hearing and slight, mild, and moderate hearing losses in young infants from ASSR data alone. Future studies are needed to investigate the accuracy of the ASSR technique for describing mild and moderate hearing losses in infants to allow for accountable and evidence-based implementation of the ASSR technique. Current clinical practice however, requires that ASSR thresholds $>60 \mathrm{~dB}$ HL in young infants be cross-checked by additional electrophysiological techniques such as the ABR.

\section{REFERENCES}

Bess, F.H., Dodd-Murphy, J., \& Parker, R.A. (1998). Children with minimal sensorineural hearing loss: Prevalence, educational performance and functional status. Ear and Hearing, 19(5), 339-54.

Carney, A.E., \& Moeller, M.P. (1998). Treatment efficacy: Hearing loss in children. Journal of Speech Language and Hearing Research, Suppl. 41, 61-84.

Clark, J.G. (1981). Uses and abuses of hearing loss classification. ASHA, 23, 493-500.

Cone-Wesson, B., Dowell, R.C., Tomlin, D., Rance, G., \& Ming, W.J. (2002). The auditory steady-state response: Comparisons with the auditory brainstem response. Journal of the American Academy of Audiology, 13, 173-187.

Davis, J.M., Elfenbein, J.L., Schum, R., \& Bentler, R. (1986). Effects of mild and moderate hearing impairments on language, educational, and psychosocial behavior of children. Joumal of Speech and Hearing Disorders, 51, 53-62.

Dimitrijevic, A., John, M.S., Van Roon, P., Purcell, D.W., Adamonist, J., Ostroff, J., Nedzelski, J.M., \& Picton, T.W. (2002). Estimating the audiogram using multiple auditory steady-state responses. Jourrial of the American Academy of Aúdiology, 13, 205-224.

Goodman, A. (1965). Reference zero levels for pure-tone audiometer. ASHA, 75, 262-263.

Gorga, M.P. (1999). Predicting auditory sensitivity from auditory brainstem response measurements. Seminars in Hearing, 20(1), 2943.

John, M. S., Brown, D.K., Muir, P.J., \& Picton, T.W. (2004). Recording auditory steady-state response in young infants. Ear and Hearing, 25(6), 539-553.

Joint Committee on Infant Hearing (2000). Year 2000 position statement: Principles and guidelines for early hearing detection and intervention programs. American Journal of Audiology, 9, 9-29.

Lins, O.G., Picton, T.W., Boucher, B.L., Durieux-Smith, A., Champagne, S.C., Moran, L.M., Perez-Abalo, M.C., Martin, V., \& Savio, G. (1996). Frequency-specific audiometry using steadystate responses. Ear and Hearing, 17, 81-96.

Lutman, M.E. (2000). Techniques for neonatal hearing screening. Seminars in Hearing, 21(4), 367-378.
Luts, H., Desloovere, C., Kumar, A., Vandermeersch, E., \& Wouters, J. (2004). Objective assessment of frequency-specific hearing thresholds in babies. International .Journal of Pediatric Otorhinolaryngology, 68, 915-926.

Luts H., \& Wouter, J. (2004). Hearing assessment by recording multiple auditory steady-state responses: the influence of test duration. Intemational Joumal of Audiology, 43, 471-478.

Perez-Abalo M.C., Savio, G., Torres, A., Martin, V., Rodriguez, E., \& Galan, L. (2001). Steady state responses to multiple amplitude modulated tones: an optimized method to test frequency specific thresholds in hearing impaired children and normal subjects. Ear and Hearing; 22, 200-211.

Picton, T.W., Durieux-Smith, A., Champagne, S., Whittingham, J., Moran, L., Giguére, C., \& Beauregard, Y. (1998), Objective evaluation of aided thresholds using auditory steady-state responses. Joumal of the American Academy of Audiology, 9, $315-331$.

Prieve, B., \& Stevens, F. (2000). The New York State universal newborn screening project: Introduction and overview. Ear and Hearing, 2I, 85-91.

Rance, G., \& Briggs, R.J.S. (2002). Assessment of hearing in infants with moderate to profound impairment: The Melbourne experience with auditory steady-state evoked potential testing. Annals of Otology, Rhinology and Laryngology; 111 Suppl 189, 22-28.

Rance, G., Dowell R.C., Rickards, F.W., Beer, D.E., \& Clark, G.M. (1998). Steady state evoked potential and behavioral hearing thresholds in a group of children with absent click evoked auditory brainstem response. Ear and Hearing, 19, 48-61.

Rance, G., \& Rickards, F. (2002) Prediction of hearing threshold in infants using auditory steady-state evoked potentials. Joumal of the American Academy of Audiology. 13, 236-245.

Rance, G., Rickards, F.W., Cohen, L.T., De Vidi, S., \& Clark, G.M. (1995). The automated prediction of hearing thresholds in sleeping subjects using auditory steady-state evoked potentials. Ear and Hearing, 16, 499- 507.

Rapin, I., \& Gravel, J, (2003). "Auditory neuropathy": Physiologic and pathologic evidence calls for more diagnostic specificity. International Journal of Pediatric Otorhinolaryngology, 67, 707-728.

Rickards, F.W., Tan, L.E., Cohen, L.T., Wilson, O.J., Drew, J.H., \& Clark, G.M. (1994). Auditory steady state evoked potentials in newborns. British .Journal of Audiology, 28, 327-337.

Sinninger, Y.S. (2002) Identification of auditory neuropathy in infants and children. Seminars in Hearing, 23(3), 193-200.

Swanepoel, D., \& Hugo, R. (2004). Estimations of auditory sensitivity for young cochlear implant candidates using the ASSR: Preliminary results. International Joumal of Audiology, 43(7), 377-382.

Swanepoel, D., Hugo, R., \& Roode, R. (2004). Auditory steady state response thresholds of children with severe to profound hearing loss. Archives of Otolaryngology Head and Neck Surgery, 130, 531-535.

Swanepoel, D., Schmulian, D., \& Hugo, R. (2004). Establishing normal hearing with the dichotic multiple frequency auditory steady state response compared to an ABR protocol. Acta Otolaryngologica, 124, 62-68.

Yoshinaga-Itano, C. (2003). Universal newborn hearing screening programs and developmental outcomes. Audiological Medicine, 1,199-206. 